\title{
Neural network approach to ECT inverse problem solving for estimation of gravitational solids flow
}

\author{
Hela Garbaa \\ Lidia Jackowska-Strumiłło \\ Krzysztof Grudzień \\ Andrzej Romanowski \\ Email: helagarbaa@gmail.com \\ lidia_js@kis.p.lodz.pl \\ kgrudzi@kis.p.lodz.pl androm@kis.p.lodz.pl
}

Lodz University of Technology, Institute of Applied Computer Science, Poland.

\begin{abstract}
A new method to solve the inverse problem of electrical capacitance tomography is proposed. Our method is based on artificial neural network to estimate the radius of an object present inside a pipeline. This information is useful to predict the distribution of material inside the pipe. The capacitance data used to train and test the neural network is simulated on Matlab using the electrical capacitance tomography toolkit ECTsim. The provided accuracy is promising and shows efficiency to solve the inverse problem in a simple manner and on reduced computational time about 120 times when compared to the existing Landweber iterative algorithm for tomographic image reconstruction that can be encouraging for dynamic industrial applications.
\end{abstract}

Index terms-Electrical Capacitance Tomography, Inverse Problem, Artificial Neural Networks, Gravitational Flow of Solids.

\section{INTRODUCTION}

$\mathrm{E}$ LECTRICAL Capacitance Tomography (ECT) is a noninvasive technique used to image the spatial distribution of merged materials with different dielectric properties inside a pipe [1]. The spatial distribution is determined by measuring the capacitances between all pairs of electrodes placed around the vessel containing the process to be examined [2]. The provided measurements depend on the electrical permittivity value of the combined materials and their spread inside the isolated pipe. Studying the relationship between capacitance records and permittivity distribution and converting them to an ECT image has been an attractive research era since 1980's $[13,15,16]$. Researchers are still investigating to improve the performances of this technique and agree on the complexity of the task due to the difficulties with inverse problem solution, nonlinearity of the system and the limited number of obtained capacitances. Typical used sensors are with $\mathrm{N}$ electrodes (e.g. $N=8,12,16$ ) lead to $M$ capacitance measurements $(M=28,66,120)$ respectively and typical generated 2D ECT image with resolu- tion of $32 \times 32$ pixels, which in turn makes the inverse problem ill-posed.

Forward and inverse problems are in fact the two main tasks in tomography visualization. First one is looking for the measurement records given permittivity distribution (in case of ECT tomography) while the later one is nothing else but looking for a relationship of the measurement (result) with the source data (cause) that is looking for the cause, which gives the measurement. In most cases, a forward problem is solved by numerical methods with the finite elements method (FEM) being the most popular one. FEM allows calculating the inter-capacitance values relying on the known permittivity distribution.

Typical results of inverse problem solution are the reconstructed images of ECT sensor space. Depending on type of the applied reconstruction algorithm the image quality can vary. Choice of appropriate reconstruction method for industrial application is limited by time of calculation in real time. In the case of iterative reconstruction methods the reconstructed image is updated iteratively until reaching a satisfactory error based on the difference between the calculated and real capacitances.

Authors in [4] reviewed various Iterative ECT methods: Newton-Raphson, Landweber iteration and Algebraic Reconstruction Techniques. Evaluation and simulations results highlighted the superiority of the performances of Landweber iterative algorithm in terms of lowest capacitance calculation errors comparing to the other cited algorithms. Artificial Neural Networks (ANN) have been used for solving both ECT problems since they represent a powerful and effective tool to dealing with complex and non-linear computations. The type of the applied neural network differs depending on the purpose of investigations. A multi-layer Feed-Forward Network (FFN) was applied to solve the forward problem. The network was trained to predict capacitance data from different permittivity distributions and then when integrated with Landweber iteration method provided a satisfactory quality of reconstructed image [3]. 313 parallel multi-layer perceptron with 2 hidden layers each 
of which were applied in [5] to reconstruct different ECT image pixels and visualize the oil distribution inside a pipe.

Large radial basis function (RBF) neural network is used in [7], with 66 nodes, representing the measured capacitances, in the input layer and 804 neurons in the output layer to flow patterns image reconstruction. The 804 outputs of the network correspond to the mesh grid elements considered to model the ECT phantoms in an earlier step.

In our present work we propose to use a Multi-Layer Perceptron with one hidden layer to estimate the radius of an object present inside the sensor and thus reconstruct the tomographic image. Our approach has the advantage to have a simple neural network with simplified structure: 66 capacitances form the input layer and one neuron at the output which corresponds to the radius of the phantom. The performance rate is set at 0.004 while it was equal to 0.3 in [7].

\section{II.ECT INVERSE PROBLEM}

Electrical capacitance tomography inverse problem is to, by means of appropriate algorithm; determine permittivity distribution based on measured capacitances into a form of a tomographic image $[10,14]$. Fig. 1 shows a schematic of an image reconstruction process in ECT
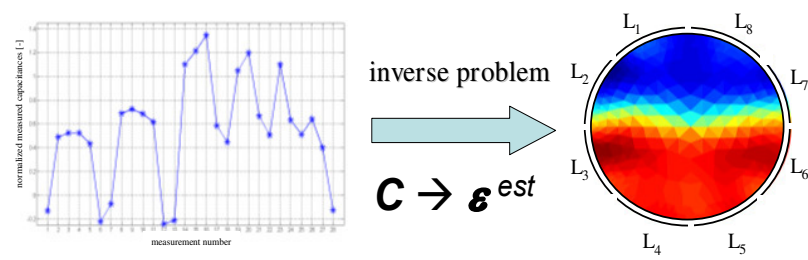

Fig. 1.Example of a graph of normalized measured capacitances and corresponding reconstructed tomography image following the solving of permittivity matrix using the inverse problem method.

Two major characteristic obstacles of inverse problem are: its ill-posed and non-linear properties. The first refers to the limited number of measured capacitances compared to the number of pixels to be reconstructed while the relationship between capacitance and permittivity distribution nonlinearity is modeled by the Gauss Law, [11, 13, 14] (Eq.1):

$$
\mathrm{C}=\frac{\mathrm{Q}}{\mathrm{V}}=-\frac{1}{\mathrm{~V}} \iint_{\Gamma} \mathrm{\varepsilon}(\mathrm{x}, \mathrm{y}) \nabla \phi(\mathrm{x}, \mathrm{y}) \mathrm{d} \Gamma_{x}
$$

where $\mathrm{Q}$ is the electric charge; $\mathrm{V}$ the potential difference between two electrodes, $\varepsilon(\mathrm{x}, \mathrm{y})$ denotes the permittivity and $\varphi(\mathrm{x}, \mathrm{y})$ represents the electrical potential distributions. $\Gamma$ stands for the electrode surface and $\mathrm{d} \Gamma$ an element orthogonal to this surface.

A discrete linear approximation of the previous equation is formulated as following (Eq. 2):

$$
\mathrm{C}=\mathbf{S} \varepsilon
$$

where $\mathbf{C}$ is a vector of measured capacitances, $\mathbf{S}$ linearized sensitivity matrix and $\varepsilon$ is a vector of permittivity distribution. The problem of image reconstruction is then reduced to solving the linear discrete form (2) which is still challenging and attracting researchers 'efforts. The problem of image reconstruction is then reduced to solving equation (3) represented:

$$
\varepsilon=S^{-1} \mathrm{C}
$$

The inverse of $\mathbf{S}$ does not exist; because $\mathbf{S}$ is not a square matrix (number of measurements is not equal of number of pixels in image). Instead of having its inverse $S^{-1}$, the pseudo-inverse $\mathbf{S}^{*}$ must be calculated, for instance, using an approximated solution. Other important problem in image reconstruction procedure is the dependence of the sensitivity matrix on the permittivity distribution, what causes that the inverse problem is non-linear. A lot of publications where different kind of methods to solve the inverse problem (linear, non-linear, directly methods and iterative methods) are presented in literature [3]. Depending on computational method, the algorithm generates images with different levels of quality, in the case of LBP algorithm or much higher quality, using iterative or non-linear methods. In the case of iterative and non-linear methods, high image quality is occupied by long computational time.

The Landweber iterative algorithm is one of the most popular methods in the field of ECT image reconstruction. The iteration process, in Landweber algorithm is governed by the following formula $[8,14]$

$$
\varepsilon_{k+1}=\varepsilon_{k}-l S^{T}\left(S \varepsilon_{k}-C\right)
$$

where $\varepsilon_{k+1}$ and $\varepsilon_{k}$ are the estimated permittivity distributions at the $k^{\text {th }}$ and $(k+1)^{\text {th }}$ iterations respectively, $\mathrm{S}$ is the calculated sensitivity matrix and $l$ is a relaxation parameter of Landweber algorithm.

The method cited above owns the advantages of easy implementation and low computational complexity but suffers from the numerical optimization point of view as it possess a relatively low convergence rate and hardly provides a global optimization solution, [12].

Artificial Neural Network constitutes a competitive optimization based- method applied in the same research era $[3,17]$. 


\section{PROBLEM STATEMENT}

The extraction of industrial process characteristic parameters gives possibility of predicting unwanted incidents and feasibility of in depth exploration of dynamic spatial temporal phenomena occurring during industrial process such as flows [13, 15, 16, 18-20]. Visualization of flow processes by means of tomography image reconstruction gives a non-invasive tool for extraction of these parameters and given sufficient reconstruction time combined with fast image processing algorithms can promise on-the-fly flow characterization.

One of the examples of successful ECT application for dynamic flow is hopper gravitational discharging process $[19,20]$. Model of such process is depicted on Fig. 2. where the laboratory setup photograph is presented. The mentioned type of flow is widely present in a range of branches such as pharmaceutical, chemical, food processing, construction and others. Processing of 2D reconstructed images allowed examination of hopper discharging funnel type of flow parameters. The set of parameters such as solids concentration in funnel and funnel area size characterize the dynamics of hopper flow [18]. This knowledge gives the information about correct/incorrect hopper flow.

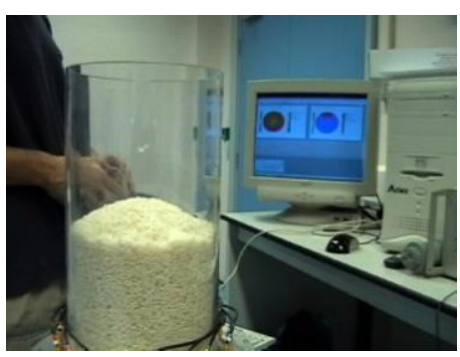

a)

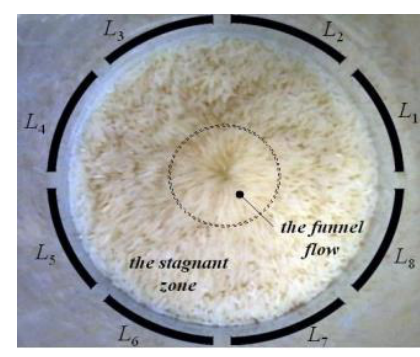

b)
Fig. 2. Photos of the hopper flow model. Photos of the hopper model: a) side view of the hopper and the image reconstruction visualisation; b) top view of the container with 8 sensor electrodes depicted around the silo.

The so-called funnel flow occurring during the silo discharging process not obvious to be analysed in its full volume since the non-transparent nature of the process. Hence, ECT is the ultimate tool to examine this flow on the base of reconstructed image where funnel area, in the center of silo, with the smaller material concentration value than rest of the sensor space can be observed (Fig. 3)

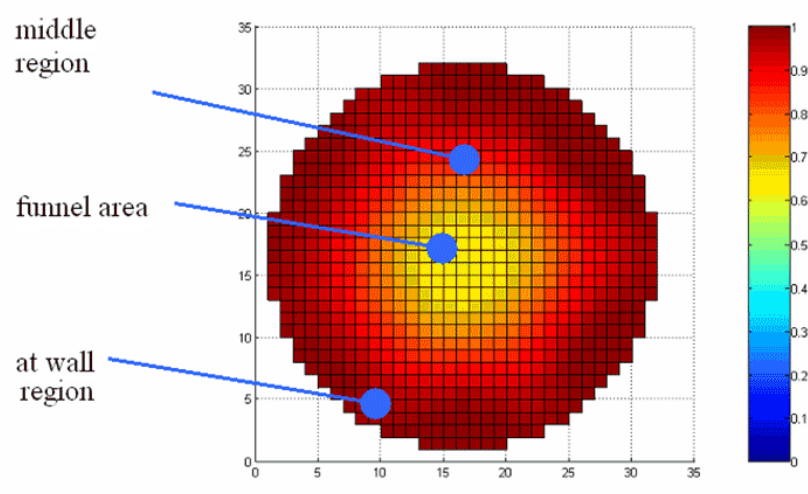

Fig. 3. Basic ECT tomographic image reconstructed for funnel hopper flow with characteristic flow areas indicated.

In order to develop the method for calculating these characteristic parameters a scheme of data processing was proposed. It allows estimating the radius of the object of a different permittivity inside a sensor cross-section space. As a proof-of-concept study we present a simulation with a phantom as useful information to predict the distribution inside the vessel knowing the capacitance measurements. A uniform distribution inside a circular sensor and a circular object is situated at the center of the vessel was assumed for calculations as shown in Fig. 4.

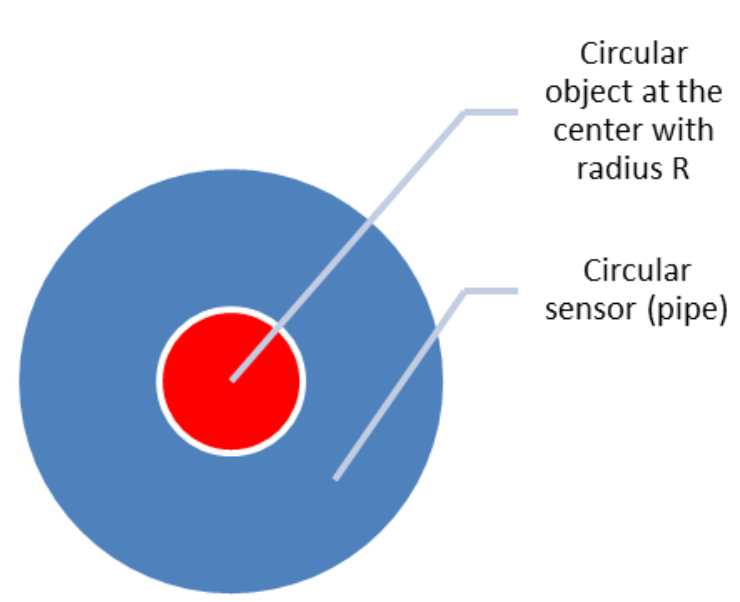

Fig. 4. Phantom considered with circular sensor and circular object in the center.

We present, in the following sections, the ECT inverse problem solving by use of Artificial Neural Networks, an evaluation of our method by comparing it to Landweber iterative approach and mention further works. 


\section{ARTIFICIAL NEURAL NETWORKS}

An artificial neural network is a powerful information processing tool recognized for its abilities to model complex input/output relationships and to learn these relationships directly from the data being modeled. They are principally designed to mimic the human brain functions in the following two ways: they acquire knowledge through a learning process, and the knowledge is stored within interneuron connection strengths known as synaptic weights, [6].

Two learning approaches are available: the first one is supervised and the second is unsupervised. A supervised learning, mostly applied, requires a desired output in order to learn. To perform the task of learning a training algorithm is applied in order to adjust the synaptic weights of the network in an orderly fashion so as to generate a model that maps the input to the output using historical data. The provided model can then be generalized to produce the output when the desired output is unknown. In addition to a powerful training algorithm, a neural network needs to have an appropriate structure to deal with the complexity of the problem to be solved. The most commonly used network structure, which has been also used in the presented approach, is the MultiLayer Perceptron (MLP). An example of a MLP network is shown in figure 5 .

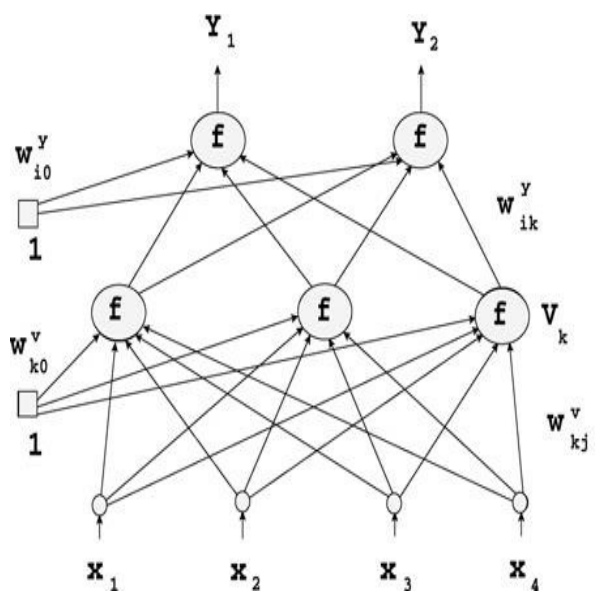

output layer

hidden layer

Fig.5. An example of multilayer perceptron network with one hidden layer.

The inputs are fed into the input layer and get multiplied by interconnection weights as they are passed from the input layer to the hidden layer. Within the hidden layer, they get summed then processed by a nonlinear function called activation function. The data is multiplied by interconnection weights then processed one last time within the output layer to produce the neural network outputs. A mathematical representation of the signal of $\mathrm{i}$-th network output $\mathrm{Yi}$ is given by the Eq. (5):

$Y_{i}=f\left(\sum_{k=0}^{M} \omega_{i k}^{Y} y_{k}\right)=f\left(\sum_{k=0}^{M} \omega_{i k}^{Y} f\left(\sum_{j=0}^{W} \omega_{k j}^{V} x_{j}\right)\right)$

Where: $\mathrm{N}-$ number of inputs, $\mathrm{M}-$ number of neurons in the hidden layer, $w_{\text {}} \mathrm{k} j$ - weight of kth neuron in the hidden layer

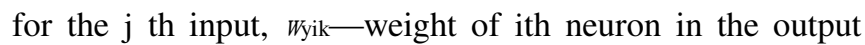
layer for the signal $\mathrm{yk}$, which is the output of the kth neuron

The activation function $\mathrm{f}(\cdot)$ may be a simple linear or a non-linear function. The most commonly used activation functions are: threshold function, sigmoidal function, hyperbolic tangent function and radial basis function. In our case we used the sigmoidal function in both hidden and output layers. Sigmoidal function is mathematically expressed as:

$$
f(z)=\frac{1}{1+e^{-z}}
$$

ANN-based inverse model is built on the basis of relations between the network input and output vectors. The knowledge about the inverse mapping is stored within the network structure and network connection weights $[6,22]$. Sixty six values of capacitances $\mathbf{C}=\left[\mathrm{C}_{1}, \ldots, \mathrm{C}_{66}\right]$ constitute the network input vector. The approximated values of the corresponding radius $\widehat{R}$ are calculated at the network output. An unknown mapping of the input vector to the output vector is approximated in an iterative procedure known as neural network training [5]. The objective of the learning algorithm is to adjust network weights on the basis of a given set of input-output pairs for a given cost function to be minimized. Back propagation algorithm, a supervised learning network algorithm, uses the gradient of the performance function to determine how to adjust the weights to minimize performance. In back propagation, the error data is propagated from the output layer backwards through the network. The effected computations allow the update of the incoming weights at each layer. In our present approach, during the network learning phase the error is propagated until a set value of the training error is reached.

\section{V.EXPERIMENTAL PART}

\section{A. Description}

We treat on our present work the inverse problem which aims to determine the material distribution relying on measured capacitances. The simulation is done using ECTSIM Matlab's toolbox, [8, 21]. ECTSIM was designed to evaluate existing image reconstruction algorithms applied on the field of ECT like Landweber algorithm and LBP method. Our work can be described on 2 main steps: (1) Sensor Modeling and capacitances measurement (2) Image Reconstruction using Artificial Neural Network: A multilayer Perceptron (MLP) is applied to determine the phantom radius). We enclose the experimental part by comparing the obtained results with our method with others obtained with Landweber algorithm. Computations were performed on PC computer with Intel(R) Xeon(R) CPU E5630 @ 2.53GHz processor and 24,0 GB RAM. The different algorithms were implemented in MATLAB.

We detail on the following paragraphs the steps mentioned above. 


\section{B. Sensor Modeling and capacitances measurement}

The current work is done under the $2 \mathrm{D}$ version of the ECTSIM toolbox using the circular sensor model. The provided sensor model is composed of four layers: pipe layer, electrode layer, insulation layer and the screen layer as shown on Fig.3. As the user of the toolbox is able to set the different parameters of each layer like thickness, electrical permittivity of insulation material and number of electrodes, we chose a sensor with 12 electrodes $(\mathrm{N}=12)$ placed inside the pipe ( inner insulator thickness $=0$ ), background permittivity $=1$ and elements' permittivity $=3$. The field of view diameter was set to $84 \mathrm{~mm}$. The sensor field of the view was then divided into $96 \times 96$ square meshes. For each phantom the simulated capacitances is calculated using the discrete linear approximation of Gauss Law (Eq.2).

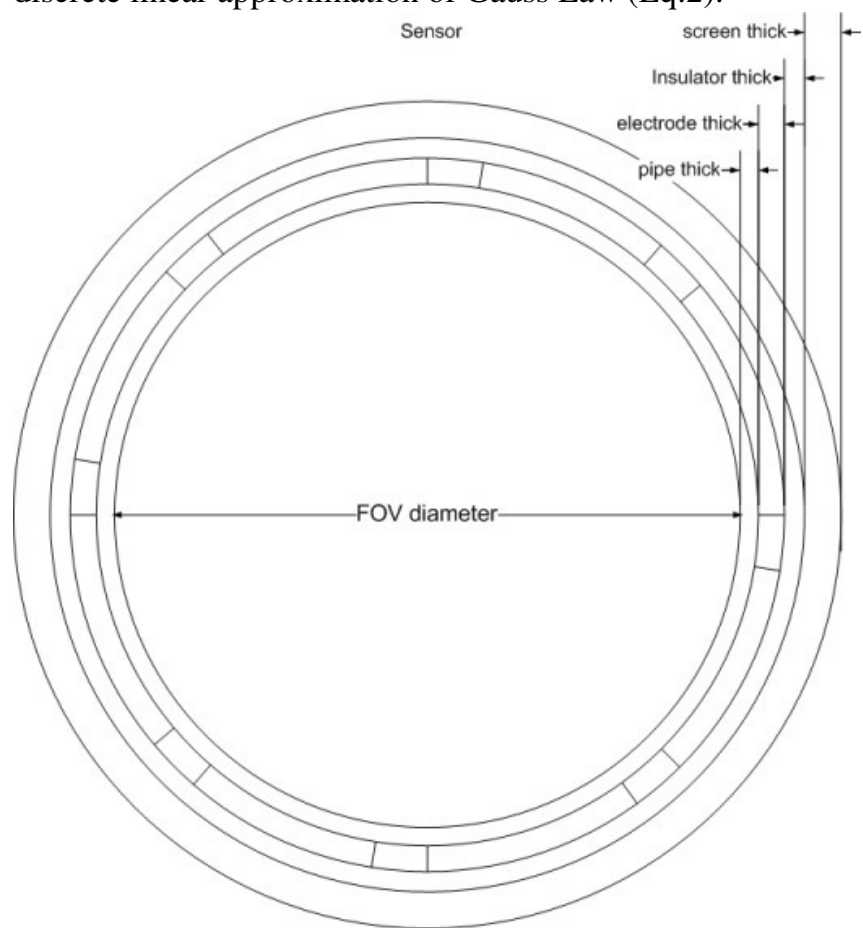

Fig.6. Sensor's diagram used for simulation [21].

We varied the diameter of the object present inside pipe in order to generate the phantoms to train and test the ANN in a farther step.

\section{Image Reconstruction using Artificial Neural Network}

For the given parameters we generated 320 phantoms divided into 241 training examples and 80 test examples. A Multi-Layer Perceptron with a single hidden layer is applied to estimate the radius of the object inside the pipe. The number of nodes in the input layer is given by the number of measured capacitances $\mathrm{L}=\mathrm{N}(\mathrm{N}-1) / 2=66$ and one neuron at the output layer. The network was trained using the back propagation algorithm with a training error set to $\mathrm{E}=$ $1 / 240=0.004$. We made several experiences to determine the number of nodes in the hidden layer. Table 1 summarizes the obtained testing errors with different numbers of hidden neurons.
We consider on the selection of the appropriate structure of MLP :

- Number of iterations at the learning phase

- Mean Square Error (MSE) during the test process:

$$
\mathrm{MSE}=\frac{1}{\mathrm{n}} \sum_{\mathrm{i}=1}^{\mathrm{n}}(R-\widehat{R})^{2}
$$

- Mean testing error:

$$
\text { Mean }=\frac{1}{\mathrm{n}} \sum_{\mathrm{i}=1}^{\mathrm{n}}\left|e_{\mathrm{i}}\right|
$$

where $\quad \theta_{i}(R)=(R-\hat{R})$

We designate by $\mathrm{R}$ the desired MLP output / desired radius, $\hat{R}$ the estimated radius at the testing phase and $n$ the number of testing examples. Table 1 summarizes the obtained testing errors with different numbers of hidden neurons.

TABLE 1.

TESTING ERRORS AND NUMBER OF ITERATIONS WITH DIFFERENT MLP STRUCTURES

\begin{tabular}{|c|c|c|c|}
\hline \multirow{2}{*}{$\begin{array}{c}\text { Network } \\
\text { structure }\end{array}$} & \multicolumn{2}{|c|}{ Testing error } & \multirow{2}{*}{$\begin{array}{c}\text { Number of } \\
\text { iterations } \\
\text { (learning) }\end{array}$} \\
\cline { 2 - 3 } & MSE & Mean & $\mathbf{1 0 8 0 8 8 5}$ \\
\hline $\mathbf{( 6 6 - 1 0 - 1 )}$ & $\mathbf{0 . 1 6 7 0}$ & $\mathbf{0 . 2 8 3 9}$ & 1419490 \\
\hline$(66-20-1)$ & 0.1688 & 0.3148 & 1815935 \\
\hline$(66-30-1)$ & 0.1721 & 0.2846 & 1225485 \\
\hline$(66-66-1)$ & 0.1832 & 0.4086 & \\
\hline
\end{tabular}

The provided results show that minimum testing errors $\mathrm{MSE}=0.1670$ and Mean $=0.2839$ are obtained for a network structure with 10 hidden neurons (66-10-1). The maximum errors were obtained with 66 neurons in the hidden layer. The use of both Mean Square Error and the Mean Error was beneficial to decide the best structure to retain for the reconstruction phase since the obtained MSE errors were close for the two first structures (66-10-1) and (66-20-1). The learning process was the fastest for the given structure and was the slowest for the structure (66-30-1) as the smaller the network structure is the less numerical complexity we have.

To consider the MLP structure (66-10-1) the appropriate structure to solve our handled problem, the process of learning and testing the selected was repeated several times and results uphold that 10 neurons in the hidden layer are sufficient to estimate the radius of the object inside the pipe.

A second attempt to evaluate the performances of the proposed method is to compare the reconstructed images from ANN with images reconstructed with Landweber algorithm under the ECTsim toolbox. 
TABLE. 2.

RECONSTRUCTED IMAGES FROM MLP AND LANDWEBER ALGORITHM

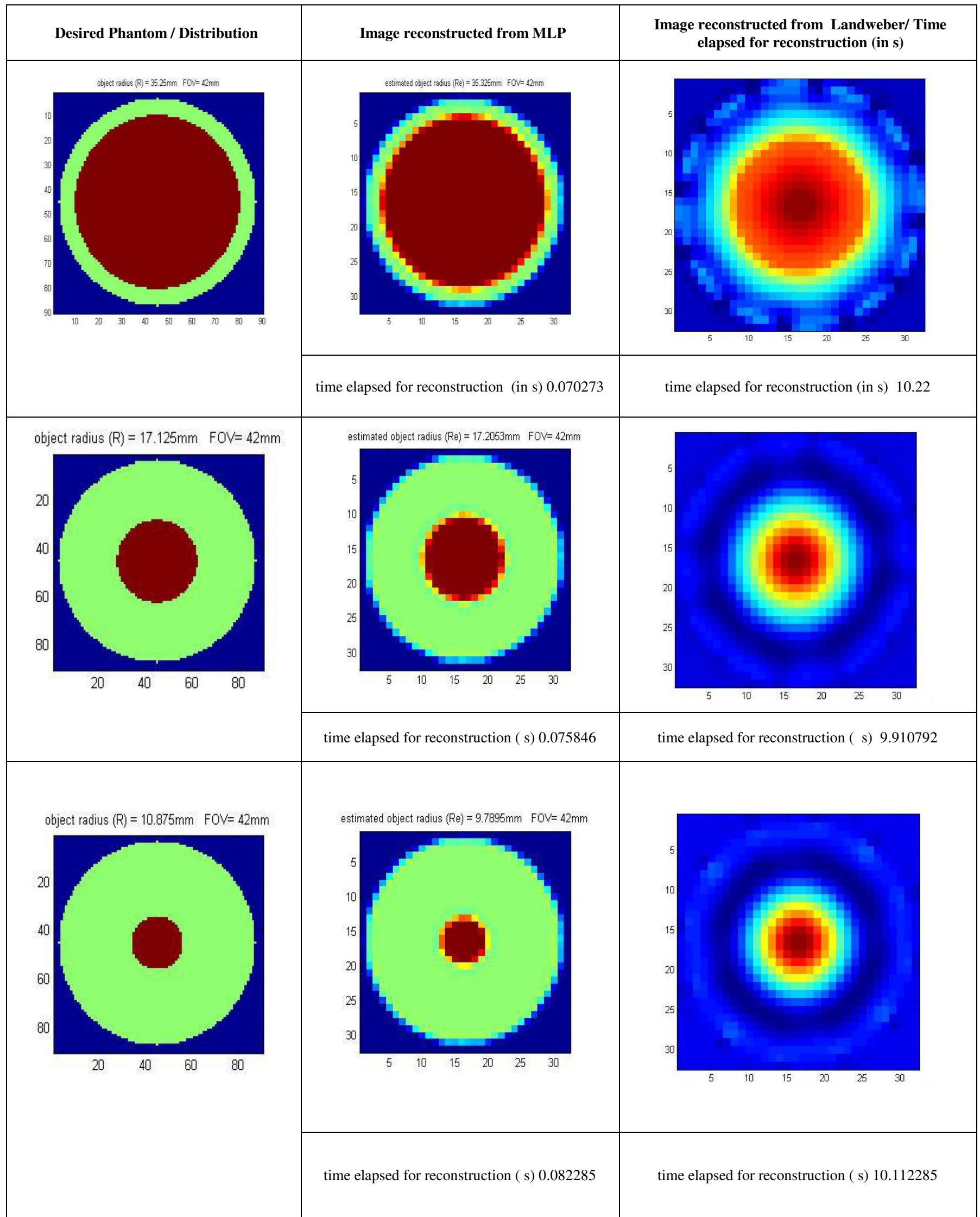


D.Evaluation of the proposed method

We present in table2. the reconstructed images from MLP network and Landweber algorithm for different radius taken from the neural network test base. The reconstruction from MLP is performed by drawing the circular object with the estimated radius. The elapsed time included one iteration to estimate the radius from the capacitances fed to the input of the MLP with the retained structure (66-10-1) and the time to draw the outer circular sensor and the object inside. The number of iterations to reconstruct the same object, using Landweber algorithm, with the same radius is set equal to 100.

The obtained images from the neural network method present satisfactory shape with small relative radius estimation error. The circular shape is better maintained with MLP based method especially in boundaries. Other advantage of the proposed methods is better estimation of permittivity value for object. In the case of MLP the relative permittivity value is much closer to the prepared simulated phantom than for Landweber algorithms. Next aspect is the time calculation. The phantom reconstruction with MLP method is about 120 times faster than with Landweber iteration algorithm.

The relative testing error for different values of radius was calculated based on formula:

$$
E_{R}=\frac{R-R}{R}
$$

where $\mathrm{R}$ is desired radius and $\hat{R}$ is the estimated radius.

The results are shown in Fig.7. The error is significant, for small values of radiuses - about $1 \mathrm{~mm}$. For $\mathrm{R}<5 \mathrm{~mm}$ the relative error value is still negative which means that the estimated value is bigger than the real value. For $5 \mathrm{~mm}<\mathrm{R}<$ $15 \mathrm{~mm}$, the relative error value becomes positive and the gap between the desired and estimated values is smaller. For radius values higher than $15 \mathrm{~mm}$ the relative testing error is near to zero. The high pic of relative error could be referred either to the capacitances measurement process (i.e. the difficulty to sense the permittivity distribution in a very small objects) or to the lack of training examples for this interval of radii. Providing more training examples for small objects $(\mathrm{R}<15 \mathrm{~mm})$ would improve the performances of our neural network.

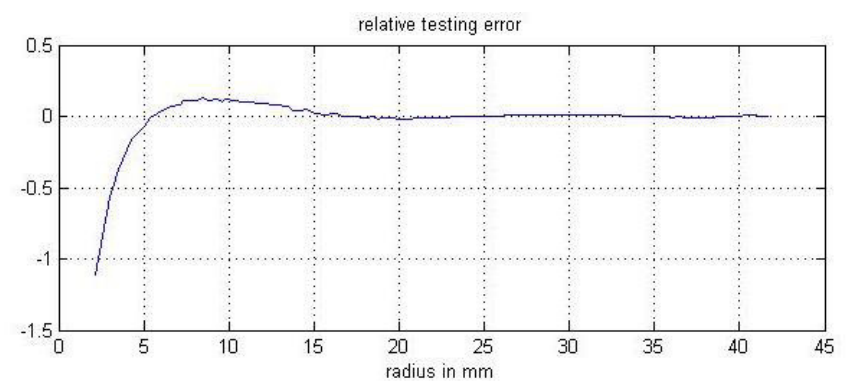

Fig. 7. Relative testing error in term of the object radius.

\section{CONCLUSION}

The aim of the work was to estimate the radius of the object present inside a circular cross section ECT sensor based on neural network technique. The calculated radius was then used to image the distribution of content inside the sensor. The different steps for solving the inverse ECT problem were depicted. The obtained results are promising especially under a simple MLP structure (66-10-1) and backpropagation training algorithm. The provided accuracy is satisfactory and ANN based approach allowed to solve the inverse problem in a simple manner and on reduced computational time about 120 times when compared to the existing Landweber iterative algorithm. Results revealed potential to estimate flow patterns such as funnel-type hopper flow with reconstruction speed sufficient for on-thefly industrial applications. Possibility to estimate more than one useful parameter (such as radius and permittivity) and generalization of the pattern of phantoms will be the subject to further work.

\section{ACKNOWLEDGMENTS}

Work partially funded by the European Commission under the Erasmus Mundus GreenIT project (GreenIT for the benefit of civil society. 3772227-1-2012-ES-ERA MUNDUS-EMA21; Grant Agreement no 2012-2625/001001-EMA2)

\section{REFERENCES}

[1] Chathurika M.G.P. Mediwaththe, Kasun E. Wijethilake, Damith B.W. Abeywardana, Sachini E. Wijethilake, Janaka V. Wijayakulasooriya, Non Invasive Cross Sectional Imaging Using Electric Capacitance Tomography, 6th International Conference on Industrial and Information Systems, ICIIS 2011, pp234-238, 10.1109/ICIINFS.2011.6038072.

[2] Norberto Flores, J Carlos Gamio, Carlos Ortiz-Alemán and Enrique Damián ,Sensor modeling for an electrical capacitance tomography system applied to oil industry, Excerpt from the Proceedings of the COMSOL Multiphysics User's Conference 2005 Boston.

[3] Qussai Marashdeh, Warsito Warsito, Liang-Shih Fan, and Fernando L. Teixeira, Nonlinear Forward Problem Solution for Electrical Capacitance Tomography Using Feed-Forward Neural Network, IEEE SENSORS JOURNAL, VOL. 6, NO. 2, APRIL 2006, pp441-449, 10.1109/JSEN.2005.860316.

[4] W Q Yang and Lihui Peng, Image reconstruction algorithms for electrical capacitance tomography, Measurement Science and Technology, Vol.44,No.1, January2003, 10.1088/09570233/14/1/201.

[5] Norberto Flores, Ángel Kuri-Morales, Carlos Gamio, An Application of Neural Networks for Image Reconstruction in Electrical Capacitance Tomography Applied to Oil Industry, Progress in Pattern Recognition, Image Analysis and Applications,Lecture Notes in Computer Science, Vol 4225, 2006, pp 371-380, 10.1007/11892755_38.

[6] L. Jackowska-Strumillo, J. Sokolowski, A. Żochowski and A. Henrot, On Numerical Solution of Shape Inverse Problems, Computational Optimization and Applications, Vo. 23, Issue 2, ,pp 231-255, 10.1023/A:1020528902875. 
[7] Jianwei Li, Xiaoguang Yang, Youhua Wang and Ruzheng Pan , An Image Reconstruction Algorithm Based on RBF Neural Network for Electrical Capacitance Tomography, Sixth International Conference on Electromagnetic Field Problems and Applications (ICEF), 2012,pp1-4, 10.1109/ICEF.2012.6310416.

[8] Smolik W and Radomski D, The matlab's toolbox for iterative image reconstruction in electrical capacitance tomography, 5th Int.Symp.on Process tomography (Poland), pp98-103.

[9] Ziqiang Cui, Chengyi Yang, Benyuan Sun, Huaxiang Wang, Liquid film thichness estimation using electrical capacitance tomography, MEASUREMENT SCIENCE REVIEW, Volume 14, No. 1, 2014, pp8-15, 10.2478/msr-2014-0002.

[10] Yunjie Yang and Lihui Peng, Data Pattern With ECT Sensor and Its Impact on Image Reconstruction, IEEE SENSORS JOURNAL, VOL. 13, NO. 5, MAY 2013, pp1582-1593, 10.1109/JSEN.2013.2237763.

[11] Jing Lei , Shi Liu , Xueyao Wang and Qibin Liu ,An Image Reconstruction Algorithm for Electrical Capacitance Tomography Based on Robust Principle Component Analysis, Sensors2013, VOL.13, pp 2076-2092, 10.3390/s130202076.

[12] Jing Lei and Shi Liu, Dynamic Inversion Approach for ElectricalCapacitance Tomography, IEEE TRANSACTIONS ON INSTRUMENTATION AND MEASUREMENT, VOL. 62, NO. 11, November2013, pp3035-3049, 10.1109/TIM.2013.2270039 .

[13] Scott DM., McCann H.: Process Imaging for automatic control, Taylor and Francis Group, p.439, 2005.

[14] Lionheart WRB.: Review: Developments in EIT reconstruction algorithms: pitfalls, challenges and recent development. Physiol. Meas., Vol. 25, 125-142, 2004, 10.1088/0967-3334/25/1/021.

[15] Yang WQ., Liu S.: Role of tomography in gas/solids flow measurement, Flow Meas. and Instrum., Vol. 11, pp. 237-244, 2000, $10.1016 /$ S09555986(00)000236.
[16] Dyakowski T., Edwards R.B., Xie C.G., Williams R.A: Application of capacitance tomography to gas-solid flows, Chemical Engineering Science, Vol.52, pp. 2099-2110, 1997, 10.1016/S00092509(97)00037-7.

[17] Warsito W. and Fan L-S (2003) Development of 3-Dimensional Electrical Capacitance Tomography Based on Neural Network Multicriterion Optimization Image Reconstruction, Proc. of 3rd World Congress on Industrial Process Tomography (Banff), 2003, 942-947.

[18] Romanowski A., K. Grudzien, R.A. Williams Analysis and interpretation of hopper flow behavior using electrical capacitance tomography Part. Part. Syst. Charact., 23 (2006), pp. 297-305, 10.1002/ppsc.200601060.

[19] Niedostatkiewicz M., Tejchman J., Chaniecki Z., Grudzień K.: Determination of bulk solid concentration changes during granular flow in a silo with ECT sensors. Chemical Engineering Science. 64, 2008, pp. 20-30.

[20] Chaniecki Z., Dyakowski T., Niedostatkiewicz M., Sankowski D.: Application of electrical capacitance tomography for bulk solids flow analysis in silos. Particle \& Particle Systems Characterization. 23, 34, 2006, pp. 306-312, 10.1002/ppsc.200601061.

[21] ECTSim 3D MATLAB's Toolbox,http://ectsim.ire.pw.edu.pl/, retrieved on April 2014.

[22] Jackowska-Strumiłło L., Sokołowski J., Żochowski A.: Topological Derivative and Training Neural Networks for Inverse Problems. In: W. Duch, J. Kacprzyk, E. Oja (Editors), et al. Artificial Neural Networks: Biological Inspirations - ICANN 2005: 15th International Conference, Warsaw, Poland, September 11-15, 2005. Lecture Notes in Computer Science, Vol. 3697/2005, Springer-Verlag GmbH, pp. 391-396, 10.1007/11550907_62. 